\title{
Optimization of the Injected Activity in Dynamic 3D PET: A Generalized Approach Using Patient-Specific NECs as Demonstrated by a Series of ${ }^{15} \mathrm{O}-\mathrm{H}_{2} \mathrm{O}$ Scans
}

Matthew D. Walker ${ }^{1}$, Julian C. Matthews ${ }^{1}$, Marie-Claude Asselin ${ }^{1}$, Azeem Saleem², Clare Dickinson ${ }^{2}$, Natalie Charnley ${ }^{2}$, Peter J. Julyan ${ }^{1,3}$, Patricia M. Price ${ }^{2}$, and Terry Jones ${ }^{2}$

${ }^{I}$ School of Cancer and Imaging Sciences, Wolfson Molecular Imaging Centre, University of Manchester, Manchester, United Kingdom; ${ }^{2}$ Academic Radiation Oncology, University of Manchester, Christie NHS Foundation Trust, Manchester, United Kingdom; and ${ }^{3}$ North Western Medical Physics, Christie NHS Foundation Trust, Manchester, United Kingdom

The magnitude of the injected activity $\left(\mathrm{A}_{0}\right)$ has a direct impact on the statistical quality of PET images. This study aimed to develop a generalized method for maximizing the statistical quality of dynamic PET images by optimizing $A_{0}$. Methods: Patient-specific noise-equivalent counts (PS-NECs) were used as a metric of the statistical quality of each time frame of a dynamic PET image. Previous methodology developed to extrapolate the NEC as a function of $A_{0}$ was extended to dynamic PET, enabling the NEC to be extrapolated as a function of both $A_{0}$ and the time after injection. This method allowed $A_{0}$ to be optimized after a single scan (at a single $A_{0}$ ), by maximizing the NEC within the time interval for which the parameter estimation is most sensitive. The extrapolation method was validated by a series of ${ }^{15} \mathrm{O}-\mathrm{H}_{2} \mathrm{O}$ scans of the body acquired in 3-dimensional mode. Each patient $(n=6)$ underwent between 3 and 6 scans at 1 bed position. The injected activities were varied over a wide range (140-840 MBq). Noise-equivalent counting rate (NECR) versus $A_{0}$ curves and the optimal injected activities were calculated from each injection. Results: PS-NECR versus $A_{0}$ curves as extrapolated from different injected activities were consistent (coefficient of variation, typically $<5 \%$ ). The optimal injected activities for an individual, as derived from these curves, were also consistent (maximum coefficient of variation, 4.3\%). For abdominal $(n=4)$ and chest $(n=1)$ scans, we found optimal injected activities of ${ }^{15} \mathrm{O}-\mathrm{H}_{2} \mathrm{O}$ in the range of 220-350 MBq for estimating blood perfusion ( $F$ ) and $660-1,070 \mathrm{MBq}$ for estimating the volume of distribution $\left(V_{T}\right)$. Higher optimal injected activities were found in the case of a pelvic scan $\left(n=1 ; 570 \mathrm{MBq}\right.$ for $\mathrm{F}$ and $1,530 \mathrm{MBq}$ for $\left.\mathrm{V}_{\mathrm{T}}\right)$. Conclusion: PS-NECs are a valid and generic method for optimizing the injected activity in PET, allowing scanning protocols to be improved after the collection of an initial, single dynamic dataset. This generic method can be used to estimate the optimal injected activity, which is specific to the patient, tracer, PET scanner, and body region being scanned.

Received Jan. 30, 2009; revision accepted May 22, 2009.

For correspondence or reprints contact: Matthew Walker, Wolfson Molecular Imaging Centre, University of Manchester, 27 Palatine Rd., Manchester, M20 3LJ, U.K.

E-mail: matthew.walker@manchester.ac.uk

COPYRIGHT $\odot 2009$ by the Society of Nuclear Medicine, Inc.
Key Words: PET; dose optimization; blood flow measurement; noise equivalent count rate; NEC

J Nucl Med 2009; 50:1409-1417

DOI: 10.2967/jnumed.109.062679

O ignificant levels of statistical noise in PET images are a common problem, especially when examining small structures or when data are acquired over a short period. The signal-to-noise ratio $(\mathrm{SNR})$ of the PET images $\left(\mathrm{SNR}_{\mathrm{IMG}}\right)$ can be maximized through optimization of the scanning protocol. Optimization of the injected activity $\left(\mathrm{A}_{0}\right)$ is of particular interest, because it has a direct impact on the image quality.

Noise-equivalent counts (NECs) are a measure of the statistical noise present in the projection data (1). NEC versus activity curves as measured in standard phantoms are commonly used to compare the performance of different PET scanners (2). NECs can also be useful for the optimization of the injected activity (3-6), scanner setup (7), and scanner design (8). By considering the statistical effects of correcting the data for unwanted random and scattered events, NECs provide a global estimate of the statistical quality of the remaining counts. The noise-equivalent counting rate (NECR) is the NEC per unit time and is given by:

$$
\mathrm{NECR}=\mathrm{T} \times \frac{\mathrm{T}}{\mathrm{T}+\mathrm{S}+\mathrm{kR}},
$$

where $\mathrm{T}, \mathrm{S}$, and $\mathrm{R}$ are the average rates of true, scattered, and random coincidences, respectively, as acquired during the given time frame and evaluated within the boundaries of the object in projection space (1). $\mathrm{k}$ is assigned the value of 1 for a noiseless randoms correction and 2 for randoms correction via the direct subtraction of delayed coincidences. The formulation of the NEC is such that the SNR of the 
non-scattered true counts is the square root of the NEC collected. In the absence of scatter and randoms, the NEC is equal to the total true coincidences.

NEC methodology can be applied to patient data to optimize scanning protocols. The relative contributions of true, random, and scattered coincidences change with activity in a manner that is dependant on many factors: the distribution of activity within the subject, the region of the body being scanned, the amount and distribution of body mass, and the design and technical performance of the PET scanner. However, if the relationship between the NECR and $\mathrm{A}_{0}$ can be estimated for a given group of subjects, one has the opportunity to optimize $\mathrm{A}_{0}$ or the scan duration.

For filtered backprojection (FBP) image reconstruction, the maximization of NEC is expected to maximize $\mathrm{SNR}_{\mathrm{IMG}}$ for most regions within the body (9). On this basis, Watson et al. $(3,10)$ developed a method to optimize $A_{0}$ that is applicable to clinical ${ }^{18} \mathrm{~F}-\mathrm{FDG}$. At the heart of this methodology is the ability to extrapolate an NECR-A $\mathrm{A}_{0}$ curve that is specific to an individual patient, using data acquired from that patient at a single injected activity. The validity of this method for an individual patient could not be fully assessed, because each subject in the study was scanned only once at a single injected activity.

This study meets 3 main aims. First, the patient-specific NECR (PS-NECR) method of Watson et al. $(3,10)$ was extended to incorporate optimization of $\mathrm{A}_{0}$ for dynamic PET. This allowed PS-NECR-versus-time curves to be extrapolated for many possible injected activities. Second, the validity of the new method was qualified in a patient study, in which 6 cancer patients were each given between 3 and 6 injections of ${ }^{15} \mathrm{O}-\mathrm{H}_{2} \mathrm{O}$ at injected activities that varied in magnitude but were similar in all other regards. Such a validation is rarely performed because it requires multiple scans of the same subject over a range of injected activities, which is not practical with ${ }^{18} \mathrm{~F}$ - or ${ }^{11} \mathrm{C}$-labeled compounds. Finally, we estimated the injected activities that were optimal for measurements of perfusion $(F)$ and the volume of distribution of water in tissue $\left(\mathrm{V}_{\mathrm{T}}\right)$, as derived from dynamic ${ }^{15} \mathrm{O}-\mathrm{H}_{2} \mathrm{O}$ scans of the body using a lutetium oxyorthosilicate (LSO)-based 3-dimensional (3D) PET/CT camera. A preliminary report of this work has been presented previously (11).

\section{MATERIALS AND METHODS}

\section{Scanner Hardware}

All scans were obtained on a Biograph-6 HiRez scanner (Siemens Molecular Imaging Inc.) (12). This is a whole-body, 3D PET/ CT scanner with LSO crystals, fast (Pico 3D) electronics, and a short coincidence timing window (4.5 ns). Images were generated using the standard manufacturer's software. This includes simulationbased scatter correction and CT-derived attenuation correction. Randoms correction was performed via the direct subtraction of delayed coincidences. After Fourier rebinning of the 3D dataset, image reconstruction was performed for each image plane by a direct inverse Fourier transform of the rebinned projection data (similar to FBP). Although iterative reconstruction (ordered-subset expectation maximization [OSEM]) was also available for this scanner, it was not used in this study because of its additional uncertainties in absolute quantification $(13,14)$.

\section{Measurement of Scanner Live-Time Functions}

All PET scanners are subject to appreciable counting losses when operating at high counting rates. This occurs as a result of detector and electronic dead-time; coincidences that would otherwise be recorded are lost. The live-time fraction characterizes this loss and can be defined as the fraction of counts that are recorded, compared with those recorded at a low counting rate. Live-time fractions are thus close to 1 at low counting rates and are less than 1 at high counting rates. The live-time fraction for a particular detector is often approximated by a function of the singles counting rate of the detector. This facilitates a correction for dead-time effects, preserving quantitation. To enable the extrapolation of patient counting rates (as in the study by Watson et al. (3)), a common live time is assumed for all detectors. We assumed that this live time, when calculated from the total singles counting rate, was independent of the object being scanned. Scanner live-time functions can then be measured in a phantom study and applied to patient data. In this study, singles were defined as detected photons that meet the energy discrimination (425-650 keV).

Whole scanner live-time functions for the Biograph were measured experimentally using the National Electrical Manufacturers Association NU 2 counting-rate phantom (2). This $20-\mathrm{cm}-$ diameter, 70-cm-long solid polyethylene phantom was centered in the 16-cm axial field of view (FOV) of the scanner. The phantom has a small (6.4-mm diameter) hole along its axial extent, at a radial offset of $4.5 \mathrm{~cm}$. A plastic tube was threaded through the phantom, into which a solution of ${ }^{11} \mathrm{C}$ was injected. After a CT scan, PET list-mode data were acquired for $300 \mathrm{~min}$ (about 15 half-lives) and rebinned into 60 frames of $5 \mathrm{~min}$. The initial activity was $943 \mathrm{MBq}$, of which approximately 20\% was within the coincidence FOV. The decaying data were used to estimate the scanner live-time functions. As in the study by Watson et al. (3), the global counting rates for singles (s), non-scattered trues (T), and randoms (R), as collected in the absence of detector dead time, are related to the activity (a) in the object by:

$$
\begin{aligned}
\mathrm{s}(\mathrm{a}) & =\left(\mathrm{c}_{\mathrm{s}} \mathrm{a}+\mathrm{s}_{\text {int }}\right) \\
\mathrm{T}(\mathrm{a}) & =\mathrm{c}_{\mathrm{T}} \mathrm{a} \\
\mathrm{R}(\mathrm{a}) & =\mathrm{c}_{\mathrm{R}}\left(\mathrm{a}+\mathrm{a}_{\text {int }}\right)^{2}
\end{aligned}
$$

$\mathrm{c}_{\mathrm{s}}, \mathrm{c}_{\mathrm{T}}$, and $\mathrm{c}_{\mathrm{R}}$ are object-specific constants. $\mathrm{s}_{\text {int }}$ represents the contribution of singles arising from the ${ }^{176} \mathrm{Lu}$ background (15), for which $a_{i n t}$ is the equivalent radioactivity required to produce this counting rate $\left(a_{\text {int }}=s_{\text {int }} / c_{s}\right)$. In reality, each of these counting rates is reduced by a live-time factor. Incorporating these factors into Equation 2 we find:

$$
\begin{aligned}
\mathrm{s}(\mathrm{a}) & =\left(\mathrm{c}_{\mathrm{s}} \mathrm{a}+\mathrm{s}_{\text {int }}\right) \times \mathrm{f}_{\mathrm{s}}[\mathrm{s}(\mathrm{a})] \\
\mathrm{T}(\mathrm{a}) & =\mathrm{c}_{\mathrm{T}} \mathrm{a} \times \mathrm{f}_{\mathrm{T}}[\mathrm{s}(\mathrm{a})] \\
\mathrm{R}(\mathrm{a}) & =\mathrm{c}_{\mathrm{R}}\left(\mathrm{a}+\mathrm{a}_{\text {int }}\right)^{2} \times \mathrm{f}_{\mathrm{R}}[\mathrm{s}(\mathrm{a})]
\end{aligned}
$$

where $f_{S}, f_{T}$, and $f_{R}$ are the live-time functions for the singles, trues, and randoms counting rates, respectively, formulated as functions of $s$. 
In the phantom experiment, the object-specific constants and $\mathrm{s}_{\text {int }}$ were calculated from linear fits to the low-counting-rate data ( $\mathrm{s}<1.3 \mathrm{Mcps}, \mathrm{a}<9 \mathrm{MBq}, 17$ frames), where Equation 2 is used (which assumes live-time fractions of 1). Equation 3 was then rearranged and used to calculate the live-time fractions for each frame, that is, over the entire range of counting rates:

$$
\begin{aligned}
& \mathrm{f}_{\mathrm{s}}[\mathrm{s}(\mathrm{a})]=\mathrm{s}(\mathrm{a}) /\left(\mathrm{c}_{\mathrm{s}} \mathrm{a}+\mathrm{s}_{\text {int }}\right) \\
& \mathrm{f}_{\mathrm{T}}[\mathrm{s}(\mathrm{a})]=\mathrm{T}(\mathrm{a}) /\left(\mathrm{c}_{\mathrm{T}}\right) \\
& \mathrm{f}_{\mathrm{R}}[\mathrm{s}(\mathrm{a})]=\mathrm{R}(\mathrm{a}) /\left[\mathrm{c}_{\mathrm{R}}\left(\mathrm{a}+\mathrm{a}_{\text {int }}\right)^{2}\right]
\end{aligned}
$$

The 3 live-time functions were estimated as functions of the singles counting rate by fitting second- or third-order polynomials to these data. Scattered coincidences (S) can be incorporated into Equations $2-4$ by assuming a counting rate-independent scatter fraction, allowing $\mathrm{T}(\mathrm{a})$ to be replaced by $(\mathrm{T}+\mathrm{S})(\mathrm{a})$. We chose this approach, modeling the scattered and non-scattered true coincidences jointly.

\section{Extrapolation of PS-NECR-A Curves}

Following the method of Watson et al. (3), the global counting rates for singles, randoms, and trues (including scatter) are extrapolated as a function of the injected activity for the specific individual, using measurements of these counting rates as obtained at a single activity. The method uses the phantom-derived livetime functions for trues, randoms, and singles counting rates.

From the measurements at a single $A_{0}$, the object-specific constants $\left(\mathrm{c}_{\mathrm{T}}, \mathrm{c}_{\mathrm{R}}\right.$, and $\left.\mathrm{c}_{\mathrm{s}}\right)$ are determined (for each time frame) by rearrangement of Equation 3. Now including scattered coincidences, this yields:

$$
\begin{aligned}
C_{\mathrm{s}} & =\frac{\left(\mathrm{s}(\mathrm{a}) / \mathrm{f}_{\mathrm{s}}[\mathrm{s}(\mathrm{a})]\right)-\mathrm{s}_{\text {int }}}{\mathrm{a}} \\
\mathrm{C}_{\mathrm{T}} & =\frac{(\mathrm{T}+\mathrm{S})(\mathrm{a})}{\mathrm{a} \times \mathrm{f}_{\mathrm{T}}[\mathrm{s}(\mathrm{a})]} \\
\mathrm{C}_{\mathrm{R}} & =\frac{\mathrm{R}(\mathrm{a})}{\left(\mathrm{a}+\mathrm{a}_{\text {int }}\right)^{2} \times \mathrm{f}_{\mathrm{R}}[\mathrm{s}(\mathrm{a})]}
\end{aligned}
$$

We assume no excretion of radioactivity from the subject, calculating a as $\mathrm{a}=\mathrm{A}_{0} \mathrm{e}^{-\lambda \mathrm{t}}$, where $\mathrm{t}$ is the time since injection and $\lambda$ is the decay constant of the radioisotope. Equation 3 can also be solved to find $\mathrm{a},(\mathrm{T}+\mathrm{S})$, and $\mathrm{R}$ as functions of the singles rate,

$$
\begin{aligned}
\mathrm{a}(\mathrm{s}) & =\frac{1}{\mathrm{c}_{\mathrm{s}}}\left[\frac{\mathrm{s}}{\mathrm{f}_{\mathrm{s}}(\mathrm{s})}-\mathrm{s}_{\text {int }}\right] \\
(\mathrm{T}+\mathrm{S})(\mathrm{s}) & =\frac{\mathrm{c}_{\mathrm{T}}}{\mathrm{c}_{\mathrm{s}}}\left[\frac{\mathrm{s}}{\mathrm{f}_{\mathrm{s}}(\mathrm{s})}-\mathrm{s}_{\text {int }}\right] \mathrm{f}_{\mathrm{T}}(\mathrm{s}) \\
\mathrm{R}(\mathrm{s}) & =\frac{\mathrm{c}_{\mathrm{R}}}{\mathrm{c}_{\mathrm{s}}^{2}}\left[\frac{\mathrm{s}}{\mathrm{f}_{\mathrm{s}}(\mathrm{s})}\right]^{2} \mathrm{f}_{\mathrm{R}}(\mathrm{s}) .
\end{aligned}
$$

These functions can be sampled at frequent intervals over a wide range of singles counting rates. Because a(s) is a monotonic function over the activities of interest, the extrapolated coincidence counting rates can be easily plotted against a or against $A_{0}$ by including a decay correction. The scatter fraction (sf), defined as $S /(T+S)$, is obtained from the scatter correction software of the scanner. The extrapolated counting rates, together with the scatter fraction, allows for the calculation of the NECR using Equation 1, with $\mathrm{k}$ assigned the value of 2 in this instance.
An estimate of the NECR is thus obtained over a wide range of injected activities, as extrapolated from measurements at a single activity. We term this an NECR-A $\mathrm{A}_{0}$ curve for the specified time frame.

All coincidence rates (and the resulting scatter fraction) used to extrapolate the NECR- $\mathrm{A}_{0}$ curves are evaluated only over the portion of the sinogram containing the object. This is determined from the attenuation correction sinogram, using a threshold of 1.1. All the required data are reported by the scatter correction software (16). Because a randoms sinogram is not normally available on this system, the randoms sinogram is assumed to be uniform and calculated from the total randoms counting rate. This assumption has been shown to be valid in the case of clinical ${ }^{18} \mathrm{~F}-\mathrm{FDG}(3)$.

\section{Generation of NECR Time Curves}

The NECR is dependent on both the distribution and the magnitude of radioactivity within an emitting object. After a bolus injection of ${ }^{15} \mathrm{O}-\mathrm{H}_{2} \mathrm{O}$ into a patient, significant changes in both the distribution and the magnitude of radioactivity occur with time. The latter is due to the radioactive decay of ${ }^{15} \mathrm{O}$ (half-life, $2 \mathrm{~min}$ ) during the course of the scan $(6 \mathrm{~min})$. The patient's NECR thus depends on both $\mathrm{A}_{0}$ and the time since injection. The dynamic PET scan cannot then be described by a point on a single NECR-A $\mathrm{A}_{0}$ curve but is instead described by a time series of points on a time series of NECR-A $\mathrm{A}_{0}$ curves. This time series is obtained by dividing the acquired patient data into short time frames and performing an NECR- $\mathrm{A}_{0}$ extrapolation on each frame individually. When combined, these NECR-A $\mathrm{A}_{0}$ curves form an NECR-A $\mathrm{A}_{0}$-time surface. Linear interpolation of this surface allows the NECR to be estimated as a function of time for any $\mathrm{A}_{0}$, providing that the singles rate remains within the range of the measured live-time functions. We term this an NECR time curve for the specified $\mathrm{A}_{0}$.

\section{Validation of Methods with Patient Data}

The counting rate extrapolation method was validated in a dose-ranging study, in which 6 cancer patients were each given a series of controlled injections of ${ }^{15} \mathrm{O}$-labeled water. We assume that the time course of the radioactivity distribution is the same for each injection but scaled in magnitude by $\mathrm{A}_{0}$. Requirements for this are minimal patient movement, no significant change in the patient state (e.g., heart rate), and a consistent method of tracer administration. The intravenous injections were controlled using an automated system (Radiowater Generator; Hidex Oy), in which each bolus $(2.5 \mathrm{~mL})$ of ${ }^{15} \mathrm{O}-\mathrm{H}_{2} \mathrm{O}$ is given reproducibly over a period of $15 \mathrm{~s}$. These injections were immediately followed by a 12.5-mL saline flush given over $75 \mathrm{~s}$. The activities administered, the patient sizes, and the scan positions are shown in Table 1. The average time between injections was $13 \mathrm{~min}$ (minimum, $10 \mathrm{~min}$ ), with PET list-mode data being acquired for $6 \mathrm{~min}$ after each injection. All injections to subject 1 were given at the back of the wrist. All other injections were given at the antecubital fossa (at the elbow), with the exception of 1 injection to subject 2, which has been discarded from this report. Ethics approval was granted by the United Kingdom's National Health Service.

For each subject, the list-mode data from each injection were rebinned into 28 frames of increasing durations $(14 \times 5 \mathrm{~s}, 5 \times 10 \mathrm{~s}$, $3 \times 20 \mathrm{~s}$, and $6 \times 30 \mathrm{~s}$ ), starting from the time of the increase in detected counts above background. These initial counts correspond to activity in the injection line, with activity arriving in the 


\section{TABLE 1. Subject Details and Injected Activities}

\begin{tabular}{|c|c|c|c|c|}
\hline $\begin{array}{c}\text { Subject } \\
\text { no. }\end{array}$ & $\begin{array}{l}\text { Weight } \\
(\mathrm{kg})\end{array}$ & $\begin{array}{c}\text { BMl } \\
\left(\mathrm{kg} \mathrm{m}^{-2}\right)\end{array}$ & $\begin{array}{c}\text { Scan } \\
\text { position }\end{array}$ & $\mathrm{A}_{0}(\mathrm{MBq})$ \\
\hline \multirow[t]{4}{*}{1} & 75 & 26 & Chest & 547 \\
\hline & & & & 558 \\
\hline & & & & 288 \\
\hline & & & & 140 \\
\hline \multirow[t]{5}{*}{2} & 61 & 25 & Pelvis & 517 \\
\hline & & & & 625 \\
\hline & & & & 740 \\
\hline & & & & 306 \\
\hline & & & & 561 \\
\hline \multirow[t]{5}{*}{3} & 54 & 23 & Abdomen & 142 \\
\hline & & & & 426 \\
\hline & & & & 302 \\
\hline & & & & 304 \\
\hline & & & & 526 \\
\hline \multirow[t]{6}{*}{4} & 53 & 19 & Abdomen & 256 \\
\hline & & & & 545 \\
\hline & & & & 523 \\
\hline & & & & 270 \\
\hline & & & & 376 \\
\hline & & & & 157 \\
\hline \multirow[t]{6}{*}{5} & 59 & 24 & Abdomen & 552 \\
\hline & & & & 504 \\
\hline & & & & 267 \\
\hline & & & & 237 \\
\hline & & & & 382 \\
\hline & & & & 172 \\
\hline \multirow[t]{3}{*}{6} & 57 & 22 & Abdomen & 508 \\
\hline & & & & 839 \\
\hline & & & & 800 \\
\hline
\end{tabular}

body approximately $15 \mathrm{~s}$ later. Zero-time was thus defined as $15 \mathrm{~s}$ after the initial increase in scanner counting rate. A second frame definition of constant frame durations throughout the whole scan (all $12 \mathrm{~s}$ ) was investigated for subjects $1-4$.

An NECR-A $A_{0}$ curve was generated from each injection and from each time frame. At each time frame, the 3-6 injections yielded different NECR-A $\mathrm{A}_{0}$ curves. These 3-6 NECR-A $\mathrm{A}_{0}$ curves provide the means for validating the NECR extrapolations. To quantitatively assess the consistency of the extrapolations at each time frame, the NECR-A $\mathrm{A}_{0}$ curves were sampled at $10-\mathrm{MBq}$ intervals between 40 and $750 \mathrm{MBq}$ (71 sampled activities). The coefficient of variation $(\mathrm{CoV}[\%], 100 \times \mathrm{SD} /$ mean) between extrapolations was calculated at each of these activities. The mean of these $71 \mathrm{CoVs}$ was then calculated, providing a measure of the spread of the extrapolated NECR-A $A_{0}$ curves for the given subject and time frame.

\section{Estimation of the Optimal Injected Activities}

The optimal $A_{0}$ 's required for estimation of $F$ and $V_{T}$ were calculated by finding the $A_{0}$ 's that led to $95 \%$ maximization of the NECR as integrated over 2 distinct time intervals: $10-70 \mathrm{~s}$ to find the optimal $A_{0}$ for estimating $F$ and $160-310 \mathrm{~s}$ for $\mathrm{V}_{\mathrm{T}}$. These data are directly extracted from the NECR-A $\mathrm{A}_{0}$-time surface. We chose the value at $95 \%$ of the maximum NEC because of the plateau-like nature of the NECR-A $\mathrm{A}_{0}$ curve; a small reduction from the peak NEC allows a large reduction in $A_{0}$. The time-integrated NECR optimization method is approximate in that it does not fully consider the sensitivity of parameter estimates to each time frame. Instead, the method assumes that the parameter estimates have equal sensitivity for all time frames within the chosen interval (and zero sensitivity to time frames outside that interval). The time intervals chosen for $\mathrm{F}$ and $\mathrm{V}_{\mathrm{T}}$ were derived from visual examination of simulated time-activity curves using a bolus-type input function, by estimating the region of the time-activity curve that was most sensitive to a given change in $\mathrm{F}$ or $\mathrm{V}_{\mathrm{T}}$, respectively.

\section{Estimation of $\mathrm{F}$ and $\mathrm{V}_{\mathrm{T}}$}

For each subject, tumor regions of interest (ROIs) were delineated on CT images and then used to generate time-activity curves from the dynamic PET data. ROI sizes ranged from 1.5 to $45 \mathrm{~mL}$ (mean, $13 \mathrm{~mL}$ ). Estimates of $\mathrm{F}$ and $\mathrm{V}_{\mathrm{T}}$ were then obtained via nonlinear least-squares minimization, using a standard 1-tissue-compartmental model (17). For subject 1, an imagederived input function was extracted from the aorta. For subjects $3-6$, an externally measured arterial input function was available, for which delay and dispersion were included in the kinetic model.

\section{RESULTS}

\section{Measurements of Scanner Live-Time Functions}

The object-independent live times as measured in the phantom study are shown in Figure 1. The singles live time is representative of count losses at the detectors. The trues and randoms live times are similar and fall at a greater rate than the singles. These coincidence live times represent the count losses at the detector, multiplexing losses within the coincidence electronics, and the application of the coincidence condition (9).

\section{Extrapolated PS-NECR-A Curves}

Examples of NECR-A $\mathrm{A}_{0}$ curves extrapolated from the 5 injections to subject 3 are shown in Figure 2. Just 2 sets of NECR-A $\mathrm{A}_{0}$ curves are shown, as generated from 2 different

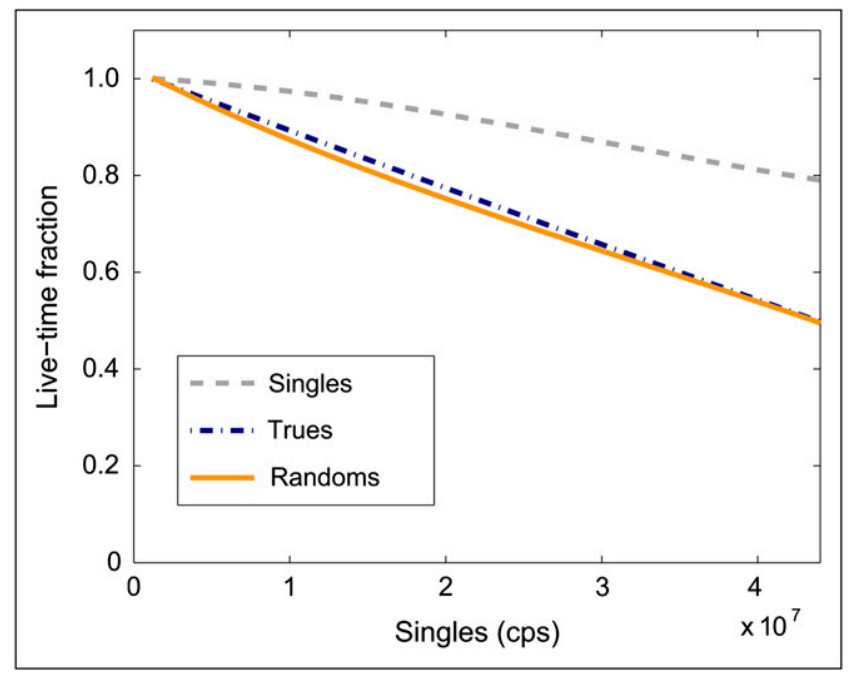

FIGURE 1. Live-time functions measured for Biograph-6 HiRez PET/CT scanner. cps = counts per second. 
time frames; 1 set is from a frame during the tracer delivery phase (27 s, important for estimates of F), and the other frame is from the tracer washout period $(255 \mathrm{~s}$, important for estimates of $\mathrm{V}_{\mathrm{T}}$ ). Within each frame, the variations between the 5 curves are small, with a similar CoV across the range of extrapolated activities. Between the 2 frames there are differences in both the maximum NECR and the value of $\mathrm{A}_{0}$ at which this maximum occurs. The curves originating from the earlier time frame in Figure 2 are more variable than those from the late time frame, as quantified in Figure 3, which shows the calculated mean CoV between the extrapolated curves as a function of the time frame from which the extrapolations pertain. These mean CoVs are calculated from 3-6 injections and as such are of low precision. The initial time frames, which cover the injection and soon after, have a larger $\mathrm{CoV}$ than do the later time frames. Once the tracer has cleared from the veins in the arm, the extrapolated curves are found to be in close agreement, with a CoV of less than 5\% for most time frames (for subjects 2-6). All injections to subject 1 were given at the back of the wrist. The NECR extrapolations, estimated scatter fractions, and time-activity curves had greater variability between injections for this subject than for the 5 subjects injected at the antecubital fossa (data not shown).

We found that the scatter correction scaling fails in the case of frames with few counts $\left(<5.5 \times 10^{5}\right.$ net trues). This problem occurred regularly near the end of the scan in the case of short (12-s) frames (present in 6/21 scans) but was not observed in the case of variable-length framing ( $30 \mathrm{~s}$ at end of scan). With the exception of these final frames, the 2

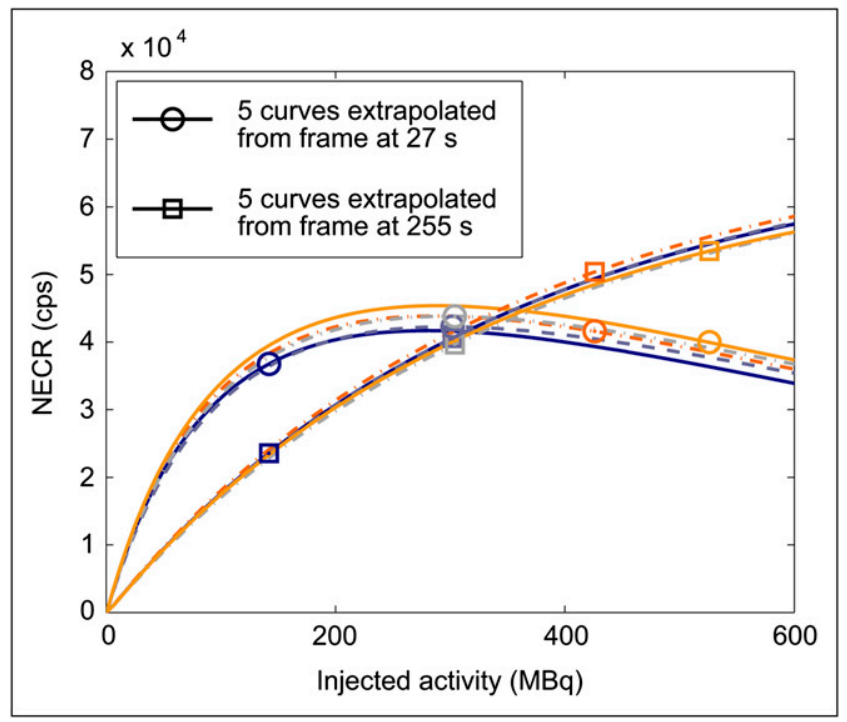

FIGURE 2. For 2 time frames, 5 extrapolated NECR-A curves for subject 3 (with $k=2$ ). The 2 sets of curves differ because of the combination of radioactive decay and change in distribution of activity within patient. Data points at 302 and $304 \mathrm{MBq}$ are overlapping. $\mathrm{cps}=$ counts per second.

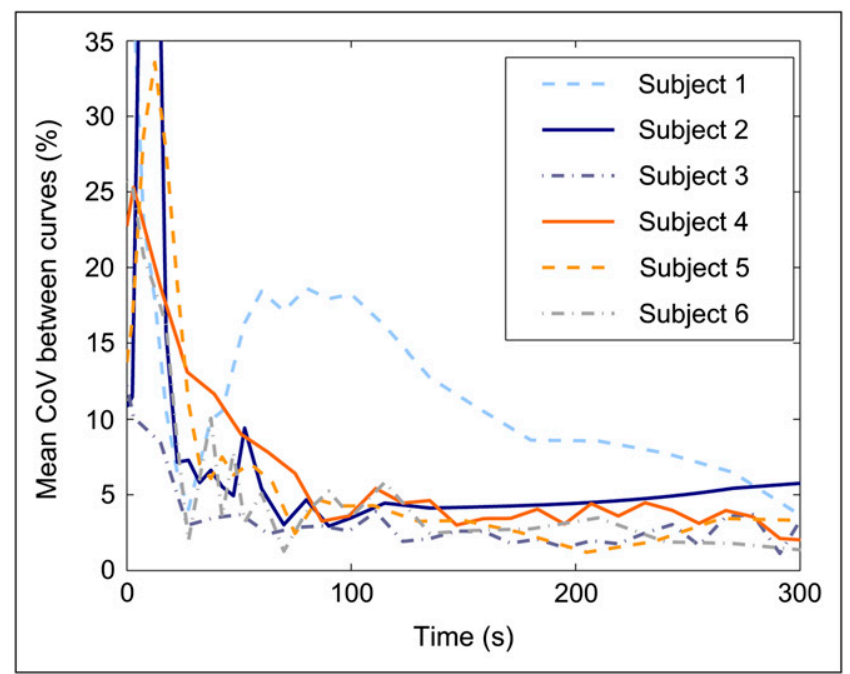

FIGURE 3. Mean CoV (\%) between several extrapolated NECR-A $A_{0}$ curves that were generated for each patient and for each time frame. CoV was averaged between 40 and 750 $\mathrm{MBq}$.

frame sequences yielded similar counting rate data and scatter fractions.

\section{Extrapolated PS-NECR Time Curves}

Examples of NECR time curves, extracted from the NECR-A $\mathrm{A}_{0}$-time surface generated for subject 4 , are shown in Figure 4. Before $25 \mathrm{~s}$, a large fraction of the activity is in the subject's arm, within the FOV. This produces a high NECR that falls as the tracer leaves the arm, before rising again when the tracer reaches the abdomen. For this subject, one sees that an $\mathrm{A}_{0}$ greater than $400 \mathrm{MBq}$ will produce little or no change in the NECR during the tracer uptake period (important for estimates of F) but that it could improve the NECR at late time frames, during tracer washout (important for estimates of $\mathrm{V}_{\mathrm{T}}$ ).

\section{Estimates of Optimal Injected Activities}

For each subject, the average NECR for the first time interval $(10-70 \mathrm{~s}, \mathrm{~F})$ is shown as a function of $\mathrm{A}_{0}$ in Figure 5A. The equivalent data for the second interval (160-310 s, $\mathrm{V}_{\mathrm{T}}$ ) is shown in Figure 5B. The presented curves are the mean of the 3-6 curves, which originate from each injection. In Figure 6, these data are used to compare the relative changes in the SNR (given by $\sqrt{\mathrm{NEC}}$ ) with $\mathrm{A}_{0}$, for which each time interval has been separately normalized to unity at the maximum SNR and for which the 4 subjects scanned at the abdomen have been averaged. The optimal $\mathrm{A}_{0}$ 's calculated from these data, that is, those that led to $95 \%$ NEC maximization, are shown in Table 2. For each subject, an optimal $\mathrm{A}_{0}$ was calculated from each injection and for each time interval $\left(\mathrm{F}\right.$ and $\mathrm{V}_{\mathrm{T}}$ ). Table 2 summarizes the mean and SD between injections for these optimal $\mathrm{A}_{0}$ 's. For abdominal $(n=4)$ and chest $(n=1)$ scans, we found optimal $\mathrm{A}_{0}$ 's of ${ }^{15} \mathrm{O}-\mathrm{H}_{2} \mathrm{O}$ in the range of $220-350 \mathrm{MBq}$ for 


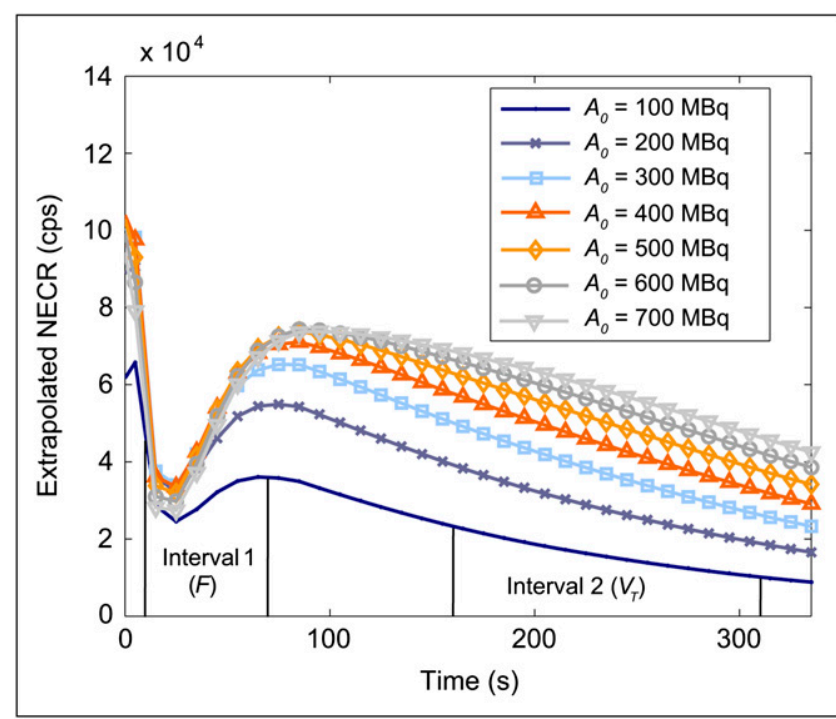

FIGURE 4. NECR time curves $(k=2)$ generated for subject 4, showing extrapolated NECR curves for different injected activities. The 2 intervals over which NECR was integrated are also shown (10-70 $\mathrm{s}$ and $160-310 \mathrm{~s})$.

estimating $\mathrm{F}$ and $660-1,070 \mathrm{MBq}$ for estimating the $\mathrm{V}_{\mathrm{T}}$. Higher optimal $\mathrm{A}_{0}$ 's were found in the case of a pelvic scan $\left(n=1 ; 570 \mathrm{MBq}\right.$ for $\mathrm{F}$ and $1,530 \mathrm{MBq}$ for $\left.\mathrm{V}_{\mathrm{T}}\right)$. The maximum $\mathrm{CoV}$ of an optimal $\mathrm{A}_{0}$ calculated from the 3-6 NECR curves for a specific subject was $4.3 \%$ (subject $1, \mathrm{~F}$ ). The mean CoVs for an individual's optimal $\mathrm{A}_{0}$ 's were $2.6 \%$ and $2.0 \%$ for the $\mathrm{F}$ and $\mathrm{V}_{\mathrm{T}}$ intervals, respectively. The optimal $\mathrm{A}_{0}$ 's for a specific subject, as derived from each injection, are thus in excellent agreement. Much larger deviations are seen to occur between different subjects.

\section{Estimates of $\mathrm{F}$ and $\mathrm{V}_{\mathrm{T}}$}

The estimates of $\mathrm{F}$ and $\mathrm{V}_{\mathrm{T}}$ are shown in Figure 7. The estimates for each tumor are separately normalized to unity to show any correlation between the parameter estimates and $\mathrm{A}_{0}$. Data from subject 2 were discarded because of difficulties in extracting an image-derived input function from the external iliac arteries. The data from the low-activity injection $(140 \mathrm{MBq})$ given to subject 1 were also discarded because the results of the kinetic fit were unstable. No significant correlation in the values of $F$ or $V_{T}$ with $A_{0}$ was observed with Pearson correlation coefficients equaling -0.18 and -0.27 . The mean estimates of $\mathrm{F}$ for the tumors analyzed here ranged from 0.34 to $0.96 \mathrm{~mL} \mathrm{~min}^{-1} \mathrm{~cm}^{-3}$, with a mean across tumors of $0.62 \mathrm{~mL} \mathrm{~min} \mathrm{mim}^{-1} \mathrm{~cm}^{-3}$. The same statistics for $\mathrm{V}_{\mathrm{T}}$ were $0.53-1.01$ and $0.84 \mathrm{~mL} \mathrm{~cm}^{-3}$, respectively.

\section{DISCUSSION}

We have shown how PS-NECR methods can be used to optimize the injected activity in dynamic PET. The data have also verified the accuracy of NECR extrapolations in the case of static imaging, in which the radiotracer is distributed throughout the subject's body. This verification comes from the good agreement between the extrapolations for all late time frames (Fig. 3). The optimal $\mathrm{A}_{0}$ 's for estimates of $V_{T}$ are 2.7-3.5 times greater than those for $F$. This is largely explained by the radioactive decay factor (2.9) between the 2 time intervals.

The variability between injections of the estimated optimal $\mathrm{A}_{0}$ 's (for a specific patient) was substantially less than the variability between subjects. Although this may cause difficulty in defining a single optimal $\mathrm{A}_{0}$ for a group of subjects based solely on their scan position and body size, examination of Figure 5 would suggest that one may inject a higher than optimal $\mathrm{A}_{0}$ without a substantial NEC penalty; conversely, if one injects a lower than optimal $\mathrm{A}_{0}$ there may be a large reduction in the average NEC. When estimating the optimal $\mathrm{A}_{0}$ for a prospective subject, it would thus be prudent to use an $A_{0}$ that is toward the top end of the measured optimal $\mathrm{A}_{0}$ 's for the given subject size and scanning position. The penalty for such an approach is that some patients would receive a small increase in the effective dose of radiation without any increase (sometimes a decrease) in the total NEC. For example, an additional 200 $\mathrm{MBq}$ of ${ }^{15} \mathrm{O}-\mathrm{H}_{2} \mathrm{O}$ will result in an increase of approximately $0.2 \mathrm{mSv}(18)$. Similarly, if one is interested in estimating both $\mathrm{F}$ and $\mathrm{V}_{\mathrm{T}}$ simultaneously, it would be reasonable to inject an activity that is greater than the optimal $\mathrm{A}_{0}$ for estimating $\mathrm{F}$ alone. Figure 6 allows the quantitative assessment of this compromise. Because the scanner used here
FIGURE 5. Average NECR-A $A_{0}$ curves for each subject, as found by integrating NECR time curve over given time interval and dividing by duration of interval. (A) Results for interval 1 (F). (B) Equivalent results for interval $2\left(\mathrm{~V}_{\mathrm{T}}\right)$. Measured values from each injection are shown as data points. Means of 3-6 extrapolated curves for each subject are shown as lines. abdom = abdomen; sub $=$ subject.
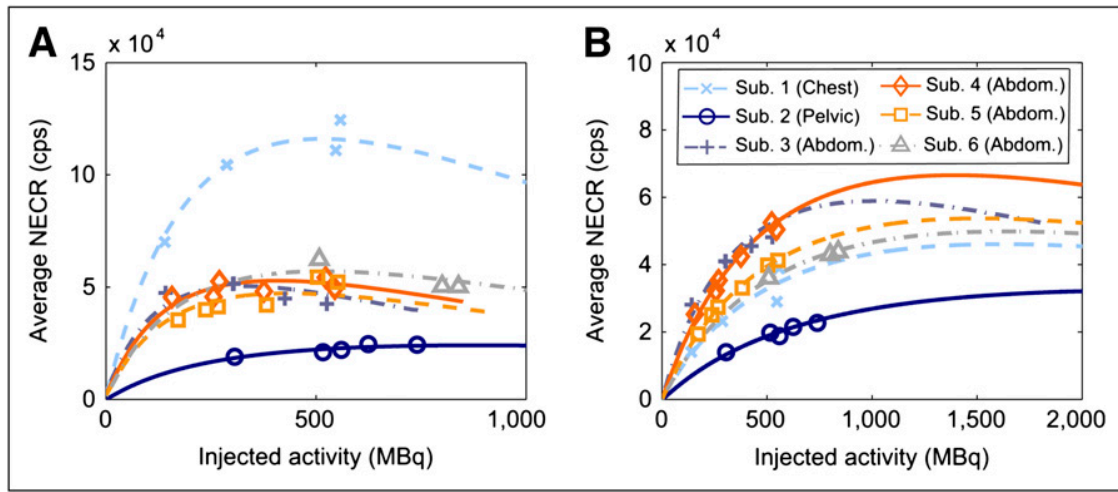


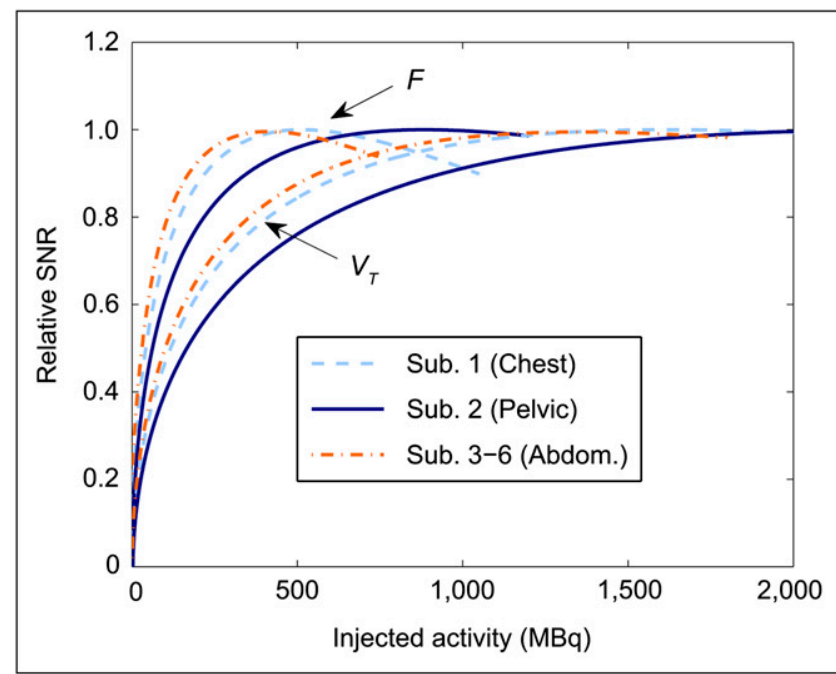

FIGURE 6. Relative SNR for 2 time intervals $\left(F\right.$ and $\left.V_{T}\right)$ and for 3 body areas scanned in this study. The 4 subjects scanned at abdomen were averaged for clarity. For each body region, curve that peaks first represents time interval 1 (F). Each time interval was separately normalized such that peak SNR is unity. sub = subject.

maintains its ability to detect and record coincidences beyond the counting rate at which the peak NEC occurs, the compromise is acceptable. The point of intersection between the 2 curves ( $\mathrm{F}$ and $\mathrm{V}_{\mathrm{T}}$ intervals) occurs within $6 \%$ of the maximum SNR in all cases.

It is worth reconsidering here the real aim of the optimization: to give more reproducible parameter estimates. Two questions arise: does NEC maximization imply maximization of the $\mathrm{SNR}_{\mathrm{IMG}}$ for the tumor, and does this maximization improve the reproducibility of the parameter estimates? The first question has been addressed by Watson, among others $(9,19)$, who has shown that for FBP reconstruction, maximization of NEC increases the local SNR for almost all regions within an image. An exception to this rule was found in the large and highactivity region of the bladder (in an ${ }^{18} \mathrm{~F}-\mathrm{FDG}$ scan), although global (95\% maximum SNR) optimization of the injected activity still resulted in SNR optimization $(>80 \%)$ for that region (9). The second question is more dependent on the methods used to estimate the parameters of interest, in particular the size of the ROI that is used to generate a time-activity curve. Increasing $\mathrm{SNR}_{\mathrm{IMG}}$ will

\begin{tabular}{ccr}
\hline TABLE 2. Estimated & $A_{0}$ for $95 \%$ NEC Maximization \\
\cline { 2 - 3 } Subject no. & \multicolumn{2}{c}{ Optimal $A_{0}(\mathrm{MBq}$ [mean $\left.\pm \mathrm{SD}]\right)$} \\
\cline { 2 - 3 } 1 & Interval $1(\mathrm{~F})$ & Interval $2\left(\mathrm{~V}_{\mathrm{T}}\right)$ \\
2 & $346 \pm 15$ & $1,050 \pm 10$ \\
3 & $568 \pm 20$ & $1,526 \pm 43$ \\
4 & $216 \pm 3$ & $661 \pm 12$ \\
5 & $268 \pm 8$ & $918 \pm 22$ \\
6 & $283 \pm 4$ & $985 \pm 11$ \\
& $339 \pm 6$ & $1,068 \pm 27$ \\
\hline
\end{tabular}

improve the precision of parameter estimates in the case in which statistical noise in the time-activity curve is a limiting factor. In our experience, this is certainly the case for small ROIs (e.g., $2 \mathrm{~mL}$ ) or in a voxelwise analysis.

Optimization methods based on the NEC do not consider the accuracy of images but simply their statistical noise. Because the injection of high activities can lead to errors in dead-time correction, care is required when determining $\mathrm{A}_{0}$. The requirement of accurate quantification in the early time frames (e.g., if using an image-derived input function) may limit $A_{0}$, preventing maximization of the NECR for the later time frames. Such problems were not apparent in our data, however. Figure 7 shows no significant correlation between the parameter estimates and the injected activity.

Optimization of $\mathrm{A}_{0}$ in the case of OSEM image reconstruction should also be considered. The presented method is valid if maximization of the NEC also maximizes $\mathrm{SNR}_{\mathrm{IMG}}$ for the ROI, that is, the tumor. For OSEM this may not be true, because unlike FBP, the regional noise in an OSEM image is strongly correlated to the activity in that region (20). The optimal $\mathrm{A}_{0}$ is then dependent on the tumor uptake. However, in the absence of a better (tumor uptakedependent) scheme for determining the optimal $\mathrm{A}_{0}$, maximization of the NEC is a reasonable approach for OSEM reconstructions. Future work is required to fully investigate the optimal $\mathrm{A}_{0}$ for OSEM reconstructions.

A possible limitation of the methodology is the use of a simple model for detector dead time. The model uses a single dead-time factor to describe dead time around the entire detector ring. This approximation may lead to errors during the initial time frames, for which most of the tracer is in the injection line and in the patient's arm. This highly asymmetric activity distribution, combined with the uncertainties in scatter correction for this distribution, may be the cause of inaccurate global counting-rate extrapolations for the initial frames. Another possible cause for the disagreement between extrapolations in the first few time frames is the variability in tracer delivery between injections. Although the rate of injection is well controlled, there is the possibility of physiologic changes between scans that could affect the blood flow (the total scanning time often exceeded $1 \mathrm{~h}$ ). With the exception of the initial time frames, the counting-rate model is found to provide accurate predictions of the NECR as a function of the injected activity throughout the dynamic scan.

For moderate-sized subjects undergoing abdominal scans on this scanner, a standard $\mathrm{A}_{0}$ of $340 \mathrm{MBq}$ of ${ }^{15} \mathrm{O}-\mathrm{H}_{2} \mathrm{O}$ is expected to produce a near-maximal NECR during the time frames that are most sensitive to changes in F. If estimating both $\mathrm{F}$ and $\mathrm{V}_{\mathrm{T}}$ from the same scan, an $\mathrm{A}_{0}$ of $600 \mathrm{MBq}$ would be reasonable in terms of NEC maximization. However, this is somewhat dependent on the aims of the investigator and the significance that is placed in each of these parameter estimates. Initial data at other bed positions indicate that the optimal $A_{0}$ for chest scans is similar to that found for abdominal scans but that pelvic scans are 
FIGURE 7. Estimates of $F(A)$ and $V_{T}$ (B), separately normalized to unity for each tumor. Data show no systematic changes in $\mathrm{F}$ or $\mathrm{V}_{\mathrm{T}}$ with injected activity. Linear regressions (gradient, intercept) are equal at reported precision $(-0.13 \times$ $\left.10^{-3}, 1.05\right)$. Results from 2 distinct metastases are shown for subject 3 . sub $=$ subject.

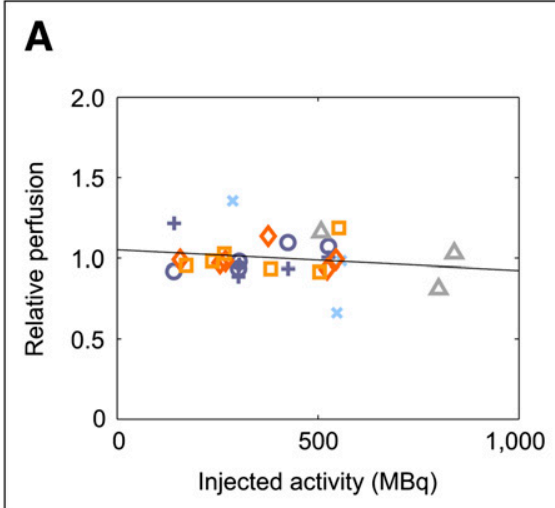

B

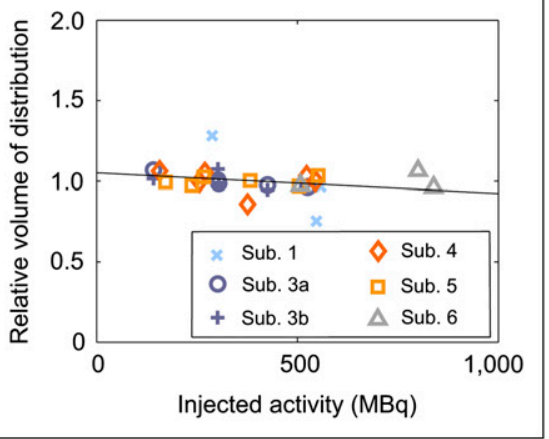

optimized at an increased $\mathrm{A}_{0}$ because of a lesser delivery and uptake of activity within the FOV.

Although multiple scans were acquired in this validation study, our data show that the optimal $\mathrm{A}_{0}$ can be estimated from a single scan for future subjects. For any patient undergoing repeated scans at the same position, it is possible to use the first scan to calculate a PS optimal $\mathrm{A}_{0}$ for the subsequent scans.

An extension to this optimization strategy would be to fully consider the biologic parameter-estimation process within the optimization rather than maximize the NEC over a representative time interval. However, the relative simplicity of the current method is one of its advantages, with the suggested extension beyond the scope of this work.

Although NEC optimization is a valid route for optimization of $\mathrm{A}_{0}$, it may be less useful for optimizing other aspects of scanning protocols, such as the site of injection. Changes in $\mathrm{A}_{0}$ do not affect the spatiotemporal distribution of the radiotracer and produce little or no change in the pattern of image variance. However, changes in the site of injection (for instance) may have a large effect on the spatiotemporal distribution of the radiotracer, which can lead to a change in $\mathrm{SNR}_{\mathrm{IMG}}$ for a given NEC and time frame (11).

\section{CONCLUSION}

PS-NECR extrapolation methods are valid when tested in human subjects and in dynamic PET. These extrapolations have been used to provide an estimate of the optimal injected activity in the case of ${ }^{15} \mathrm{O}-\mathrm{H}_{2} \mathrm{O}$ PET scans of the body, acquired in 3D mode.

The results of this work can guide future refinement and optimization of scanning methods and protocols for ${ }^{15} \mathrm{O}-$ $\mathrm{H}_{2} \mathrm{O}$ scans on modern whole-body LSO-based PET cameras. The generic method of optimizing the injected activity may also be applied to other scanners and radiotracers.

\section{ACKNOWLEDGMENTS}

We thank Charles Watson, Bernard Bendriem, Lars Eriksson, and Hartwig Newiger of Siemens Medical
Solutions for their useful comments and support. We also thank the operational staff at WMIC for their help in data acquisition, Rainer Hinz for many helpful discussions, and Juan Valle for his help in patient recruitment. This work was supported in part by a studentship from Siemens Medical Solutions and was funded in part by Cancer Research U.K. (grant 153/A4331) and the U.K. Department of Health (Experimental Cancer Centre grant).

\section{REFERENCES}

1. Strother SC, Casey ME, Hoffman EJ. Measuring PET scanner sensitivity: relating countrates to image signal-to-noise ratios using noise equivalents counts. IEEE Trans Nucl Sci. 1990;37:783-788.

2. National Electrical Manufacturers Association. NEMA Standards Publication NU 2-2001. Performance Measurements of Positron Emission Tomographs. Rosslyn, VA: National Electrical Manufacturers Association; 2001.

3. Watson CC, Casey ME, Bendriem B, et al. Optimizing injected dose in clinical PET by accurately modeling the counting-rate response functions specific to individual patient scans. J Nucl Med. 2005;46:1825-1834.

4. Danna M, Lecchi M, Bettinardi V, et al. Generation of the acquisition-specific NEC (AS-NEC) curves to optimize the injected dose in $3 \mathrm{D}{ }^{18} \mathrm{~F}-\mathrm{FDG}$ whole body PET studies. IEEE Trans Nucl Sci. 2006;53:86-92.

5. Lartizien C, Comtat C, Kinahan PE, Ferreira N, Bendriem B, Trebossen R. Optimization of injected dose based on noise equivalent count rates for 2- and 3-dimensional whole-body PET. J Nucl Med. 2002;43:1268-1278.

6. Badawi RD, Marsden PK, Cronin BF, Sutcliffe JL, Maisey MN. Optimization of noise-equivalent count rates in 3D PET. Phys Med Biol. 1996;41:1755-1776.

7. Surti S, Karp JS. Imaging characteristics of a 3-dimensional GSO whole-body PET camera. J Nucl Med. 2004;45:1040-1049.

8. Eriksson L, Townsend D, Eriksson M, et al. The NEC dependence of different scintillators for positron emission tomography. IEEE Nucl Sci Symp Conf Rec. 2004;6:3785-3788.

9. Watson CC. Count rate dependence of local signal-to-noise ratio in positron emission tomography. IEEE Trans Nucl Sci. 2004;51:2670-2680.

10. Watson CC, Casey ME, Beyer T, Bruckbauer T, Townsend DW, Brasse D. Evaluation of clinical PET count rate performance. IEEE Trans Nucl Sci. 2003;50:1379-1385.

11. Walker MD, Matthews JC, Asselin M-C, Saleem A, Price P, Jones T. Patientspecific noise-equivalent-counts from repeated, dose varying [O-15] $\mathrm{H}_{2} \mathrm{O}$ PET scans. IEEE Nucl Sci Symp Conf Rec. 2007;5:3277-3282.

12. Brambilla M, Secco C, Dominietto M, Matheoud R, Sacchetti G, Inglese E. Performance characteristics obtained for a new 3-dimensional lutetium oxyorthosilicate-based whole-body PET/CT scanner with the National Electrical Manufacturers Association NU 2-2001 standard. J Nucl Med. 2005;46:20832091.

13. Bélanger M-J, Mann JJ, Parsey RV. OS-EM and FBP reconstructions at low count rates: effect on 3D PET studies of $\left[{ }^{11} \mathrm{C}\right]$ WAY-100635. Neuroimage. 2004;21:244-250.

14. Boellaard R, Van Lingen A, Lammertsma AA. Experimental and clinical evaluation of iterative reconstruction (OSEM) in dynamic PET: quantitative characteristics and effects on kinetic modeling. J Nucl Med. 2001;42:808-817. 
15. Eriksson L, Watson CC, Wienhard K, et al. The ECAT HRRT: an example of NEMA scatter estimation issues for LSO-based PET systems. IEEE Trans Nucl Sci. 2005;52:90-94.

16. Watson CC, Casey ME, Michel C, Bendriem B. Advances in scatter correction for 3D PET/CT. IEEE Nucl Sci Symp Conf Rec. 2004;5:3008-3012.

17. Kety SS. The theory and applications of the exchange of inert gas at the lungs and tissues. Pharmacol Rev. 1951;3:1-41.
18. Brihaye C, Depresseux J-C, Comar D. Radiation dosimetry for bolus administration of oxygen-15-water. J Nucl Med. 1995;36:651-656.

19. Pajevic S, Daube-Witherspoon ME, Bacharach SL, Carson RE. Noise characteristics of 3-D and 2-D PET images. IEEE Trans Med Imaging. 1998;17:9-23.

20. Riddell C, Carson RE, Carrasquillo JA, et al. Noise reduction in oncology FDG PET images by iterative reconstruction: a quantitative assessment. J Nucl Med. 2001;42:1316-1323. 